\title{
Tenure de la terre, tenure de l'eau : enquête ethnographique dans le centre de la plaine de la Beqaa (Liban)
}

\author{
Auteurs : R. Antonius, J. Trottier
}

Adresse : Rachad Antonius : Sociologie, Université du Québec à Montréal, CP 8888, Succursale Centre-Ville, Montréal, QC, Canada, H3C 3P8

Résumé:

Cette enquête ethnographique conduite en 2015 vise à comprendre comment les agriculteurs de la Beqaa construisent leur tenure de l'eau d'irrigation en lien avec la tenure de la terre, et quelles sont les modalités d'appropriation et d'utilisation de cette eau en fonction de son origine (source, rivière, ou puits) et des moyens de sa distribution (gravité, pompes, goutte-à-goutte). Elle s'est déroulée dans le village de Qoubb Elias, où coulent les sources de Qoubb Elias et de Kherayzat, ainsi que dans le village d'Anjar, toutes localités situées au centre de la plaine de la Beqaa, et cela dans une perspective comparative avec huit autres terrains différents à travers le monde. Nous nous sommes inspirés de la théorie de la critical political ecologyqui éclaire la façon dont les facteurs sociaux et politiques structurent le rapport des acteurs aux ressources naturelles et orientent les options qui s'offrent à eux. Au-delà du constat désormais classique que les facteurs économiques et géostratégiques amènent les agriculteurs à modifier les modalités de leur accès à l'eau et à la terre, nous dégageons quatre cas de figure qui illustrent comment ces divers facteurs interagissent pour façonner différents usages des moyens techniques (pompes, puits, irrigation par immersion ou par goutte-à-goutte).

Mot-clés: tenure foncière, tenure de l'eau, transformation agricole, irrigation, Liban

\begin{abstract}
:
This ethnographic research, conducted in 2015, aimed at understanding how farmers in the Beqaa plain in Lebanon constructed their tenure of irrigation water in relation to land tenure, and what were the modalities of their appropriation of water and their use of it, as a function of the origin of the water (source, river or well) and of the ways it was distributed (gravity, pumps, or drip). The field research has been conducted in Qubb Elias, where the two sources of Qubb Elias and Kheyrazat flow, and in the village of Anjar, in the central Beqaa, in a comparative perspective in eight other locations across the world. We were inspired by the theory of critical political ecology which casts light on the way social and political factors structure the agents' understanding of their relationship with natural resources and of the options that are offered to them. Beyond the now classical statement that economic and geostrategic factors are responsible for leading farmers into changing the ways they access water and land, we have identified four typical cases which illustrate how economic, social and geographical factors interact to produce various modalities of dealing with technical means (pumps, wells or flood or drip irrigation).
\end{abstract}

Keywords: land tenure, water tenure, agricultural transformation, irrigation, Lebanon 


\section{Introduction}

La littérature scientifique étudie généralement la tenure foncière indépendamment de la tenure de l'eau. On définit la tenure d'une ressource comme la relation légale ou coutumière entre des humains, en tant qu'individus ou en tant que groupes, par rapport à une ressource (Hodgson, 2016). La tenure peut donc être formelle ou informelle, individuelle ou collective. Elle peut s'appliquer aussi bien à l'eau qu'à la terre ou à d'autre ressources comme les pêcheries ou les forêts. Dans le cas d'une terre irriguée, la tenure foncière est coproduite en interaction avec la tenure de l'eau (Trottier et al., 2019a). Cependant, le processus de construction de la tenure de la terre ne recouvre que partiellement celui de la construction de la tenure de l'eau.

La tenure foncière est nécessairement coproduite en interaction avec la représentation de l'espace (Le Roy, 2011). Le Roy reprend plusieurs types de représentations de l'espace issues de la géomatique française. Cette étude n'en reprendra que deux: la représentation géométrique et la représentation topocentrique. Au sein d'une représentation géométrique, l'espace est mesuré en fonction de coordonnées et peut être cartographié dans son ensemble. Il s'agit de la représentation sur laquelle se base un cadastre, par exemple. Au sein d'une représentation topocentrique, l'espace est lié à un point particulier et organisé autour de ce point. Ce point peut être le lieu où s'exerce une fonction politique, économique ou religieuse.

La tenure de l'eau, en revanche, a longtemps été coproduite en interaction avec le flux qu'est l'eau, le long de sa trajectoire. On peut distinguer trois types de trajectoires pour l'eau: spatiale, institutionnelle et sectorielle (Trottier et al., 2019b). La trajectoire spatiale de l'eau décrit le parcours de l'eau à travers l'espace. La trajectoire institutionnelle de l'eau décrit le parcours de l'eau à travers les diverses institutions qui la gèrent le long de cette trajectoire spatiale. La trajectoire sectorielle de l'eau décrit le parcours de l'eau à travers les différents types d'usage qui en sont faits successivement. Toutes ces trajectoires décrivent le parcours de l'eau entre le moment où elle émerge de la terre ou de la station de dessalement d'eau de mer et le moment où elle quitte le système soit en s'évaporant, soit en étant transpirée par une plante ou un animal, soit en rejoignant la mer. La tenure foncière fut longtemps élaborée très localement, réglementant l'interaction avec l'eau entre le point où la source émergeait du sol et le point où l'eau était transpirée par la plante, dans le lopin de terre où un chevelu d'irrigation avait acheminé l' eau par gravité.

La plaine de la Beqaa a longtemps fait partie de l'Empire ottoman, une période qui a connu de nombreux déplacements de population, avec les plaines peu cultivées car vulnérables aux attaques bédouines, contrairement aux zones de montagne (de Planhol, 1968). Cette vulnérabilité se maintient jusqu'à la toute fin du 19e siècle (Granott, 1952). Les populations qui s'y installent au $19^{\mathrm{e}}$ siècle le font collectivement et développent entre elles leurs propres règles de tenure foncière et de tenure de l'eau au moment même où elles développent l'irrigation.

Au cours de la seconde moitié du $20^{e}$ siècle, l'Empire ottoman tente d'appliquer le Mejelle un code de 1858 qui réglementait le foncier - afin de pouvoir augmenter la production agricole et les impôts afférents. Au Levant, les paysans ont beaucoup résisté à l'application de cette mesure, malgré la nécessité d'inscrire la terre qu'ils cultivaient à leur nom en tant 
que propriété privée. Cette résistance a souvent été expliquée comme une réticence des paysans à payer leurs taxes. Cette hypothèse n'est cependant pas validée par les travaux d'Abdulfattah qui spatialisa les résultats des quatre recensements ottomans réalisés au cours du $16^{\mathrm{e}}$ siècle dans le territoire situé au sud de la Beqaa (Hutteroth and Abdulfattah, 1977). Les paysans payaient leurs taxes en fonction de leur production agricole, quel que soit le statut de leur tenure foncière. Il est peu probable que les paysans vivant dans le territoire actuel du Liban et particulièrement la plaine de la Beqaa aient bénéficié d'un autre statut . La réticence des paysans à inscrire leurs terres à leur nom en tant que propriété privée s'explique probablement par leur endettement très élevé au cours de la deuxième moitié du $19^{e}$ siècle et jusqu'à la première moitié $20^{e}$. Étant donné que la terre ne leur appartenait pas, elle ne pouvait pas leur être confisquée en tant que garantie collatérale d'une dette. Alternativement, les pratiques de tenure collective sécurisaient grandement la paysannerie.

La loi libanaise qui réglemente le foncier aujourd'hui fut adoptée en 1930 (Hamadeh et al. 2015). Cette loi consolide le régime de propriété de la terre agricole qui devient un bien commercial pouvant être acheté, vendu ou loué. Le Liban, en revanche, ne réglemente pas simultanément l'eau d'irrigation. Tantôt fournie gratuitement, tantôt vendue et achetée, ou encore fournie gratuitement, mais livrée aux champs contre paiement, les " propriétaires » ou les détenteurs légitimes de cette ressource hydrique ne sont pas toujours clairement déterminés. Les règles qui guident sa répartition entre usagers et sa circulation ne sont pas clairement établies, faisant ainsi l'objet de négociations permanentes et de solutions alternatives et consensuelles entre acteurs locaux.

L'origine de l'eau d'irrigation dans la plaine de la Beqaa a toujours été multiple: pluie, sources, ou puits artésiens. Cette variété tend à multiplier les modes de tenure locaux qui ont été élaborés. En parallèle à la tenure locale de l'eau, le concept de l'eau «virtuelle », érigé à l'échelle des pays consommateurs d'eau, a propagé la thèse selon laquelle les pays pauvres en eau préfèrent importer les denrées agricoles plutôt que de consommer leurs maigres ressources en eau pour les produire eux-mêmes. Allan (1997) défend depuis longtemps la thèse selon laquelle ces pays gagnent à utiliser leur eau pour générer une plus grande valeur ajoutée et importer les denrées nécessaires à leur sécurité alimentaire. Plusieurs publications ont proposé des calculs de flux d'eau virtuelle entre pays exportateurs et importateurs de denrées alimentaires (Hoekstra et al., 2011). Les recherches récentes invalident cependant les hypothèses avancées par le concept d'eau virtuelle. Le calcul des volumes d'eau virtuelle tient uniquement compte de variables agronomiques et climatiques, à une échelle régionale et globale. L'identification des pays qui bénéficient d'importer de l'eau virtuelle s'effectue quant à elle uniquement en référence à une variable pluviométrique et démographique à l'échelle nationale. Cependant, les recherches récentes sur les usages de l'eau à une échelle plus locale ont démontré que les variables sociales et économiques déterminent le contenu d'eau virtuelle d'une récolte, et donc l'utilité d'importer ou d'exporter de l'eau virtuelle, bien plus que les variables climatiques et agronomiques (Trottier and Perrier, 2017).

La plaine de la Beqaa (بقاع) se situe à l'est du Liban, coincée entre deux chaînes montagneuses, entre 800 et 900 mètres d'altitude. D'une longueur de $120 \mathrm{~km}$ et d'une largeur fluctuante entre 8 et $15 \mathrm{~km}$, elle reçoit en moyenne $700 \mathrm{~mm}$ de pluie au sud, et près de $200 \mathrm{~mm}$ au nord. 
L'Oronte et le Litani sont les deux rivières principales qui l'irriguent et drainent l'eau (Universalis.fr, 2019) ${ }^{1}$.

Depuis la fin de la guerre du Liban en 1990, le paysage hydro-foncier a beaucoup changé. La concentration des terres s'est accrue. 1,6\% des exploitations font plus de 10 hectares et constituent 30\% des surfaces cultivées. La surface agricole a quintuplé entre 1961 et 1999. Le forage de puits artésiens s'est multiplié. L'économie agricole s'est libéralisée et s'est orientée vers l'exportation à destination des marchés de la péninsule arabique à travers des produits à haute valeur ajoutée. Or, près de $66 \%$ des terres agricoles irriguées du Liban se situent dans la Beqaa. Au cours des quarante dernières années, légumes et arbres fruitiers ont remplacé la culture du blé, entraînant une demande accrue en eau.

La plaine de la Beqaa nous offre donc un exemple de transformation de la tenure de la terre et de l'eau qui mérite analyse. La question générale qui se pose porte sur les facteurs sociaux qui déterminent, ou du moins affectent, les modalités de circulation et l'usage de l'eau d'irrigation. Des questions plus spécifiques se posent alors: est-ce que les choix d'assolement, qui ont un impact direct sur l'usage de l'eau, sont déterminés surtout par des facteurs sociaux et économiques ou hydrographiques ? Quelle est la place de la structuration des marchés agricoles, au niveau local et régional, et de la disponibilité de la main-d'œuvre ? Répondre à ces questions implique d'étudier simultanément la construction de la tenure de l'eau et celle de la terre afin de comprendre comment elles se co-construisent mutuellement. A priori, on pourrait formuler l'hypothèse selon laquelle les conditions géographiques et climatiques priment : origine de l'eau (pluie, puits artésiens ou sources et rivières), degré de sécheresse, quantité disponible, et facilité d'accès. Mais nous verrons dans les témoignages de nos répondants comment ces divers facteurs sont intervenus dans leurs prises de décisions.

\section{Méthodologie de l'enquête de terrain}

L'article s'appuie sur une enquête ethnographique qui s'est déroulée en 2015 dans le village de Qoubb Elias où se situe la source du même nom, dans la localité de Kherbet Qanafar où se situe la source de Kherayzat, ainsi que dans le village de Anjar, irrigué par les rivières Ghozeil et Chamsine qui prennent leur eau de la source Anjar. Ces villages situés au centre de la plaine de la Beqaa ont fait l'objet d'une analyse comparative avec huit autres terrains dispersés à travers le monde (Figure 1$)^{2}$. Le projet explorait les rapports entre tenure de la terre et tenure de l'eau, en lien avec les transformations structurelles des marchés agricoles. Nous retiendrons ici les comparaisons qui émergent avec le terrain palestinien, qui partageait jusqu'à la fin de la Première Guerre mondiale une histoire similaire à celle de la plaine de la Beqaa.

\footnotetext{
1 Voir aussi la thèse de doctorat de Roland Riachi (Riachi, 2006) pour une discussion plus approfondie et globale des questions de l'eau au Liban.

2 Dirigé par Julie Trottier, le projet De terres et d'eaux, financé par l'Agence Nationale de la Recherche, étudia les stratégies d'adaptation des petits paysans face aux bouleversements du foncier, de la tenure de l'eau et du changement climatique dans huit pays différents, incluant le Liban et les Territoires Palestiniens.
} 
Pour cela, nous avons visité sept sites différents dans ces trois villages du centre de la Beqaa, et mené des entretiens libres avec une vingtaine d'agriculteurs et de responsables (dont le maire de l'une des municipalités), en précisant bien qu'il s'agissait d'une recherche académique concernant la gestion de l'eau d'irrigation, qui ferait l'objet d'une publication. Les discussions se sont déroulées entièrement en langue arabe, quelquefois de façon individuelle, et quelquefois de façon collective avec 2, 3 ou 4 participants. Nous n'avons pas utilisé de questionnaire ni de grille rigide de questions semi-dirigées, mais avons demandé aux agriculteurs de raconter l'histoire de leur terre et de ses besoins en eau, en posant, à l'occasion, des questions spécifiques sur les changements de cultures, sur les besoins en eau de chaque culture, et sur la façon dont ils s'y prenaient pour répondre à ces besoins compte tenu de la disponibilité de cette ressource. C'est ainsi que nous avons pu obtenir un matériel très riche qui évoquait des trajectoires familiales, des considérations sur les marchés agricoles locaux et régionaux, sur les contextes politiques fluctuants, ainsi que sur les variations annuelles de pluviométrie et les techniques d'irrigation. Nous n'avons pas enregistré les discussions et n'en avons donc pas fait de transcriptions verbatim. Mais nous avons pris de nombreuses notes, que nous complétions après coup si nécessaire. Les quatre cas de figure dont nous parlerons plus bas ont émergé de notre analyse du terrain et ne constituent pas une variable déterminée $a$ priori avant l'enquête. Nous avons complété les informations sur les disponibilités en eau avec des recherches dans la littérature scientifique. 


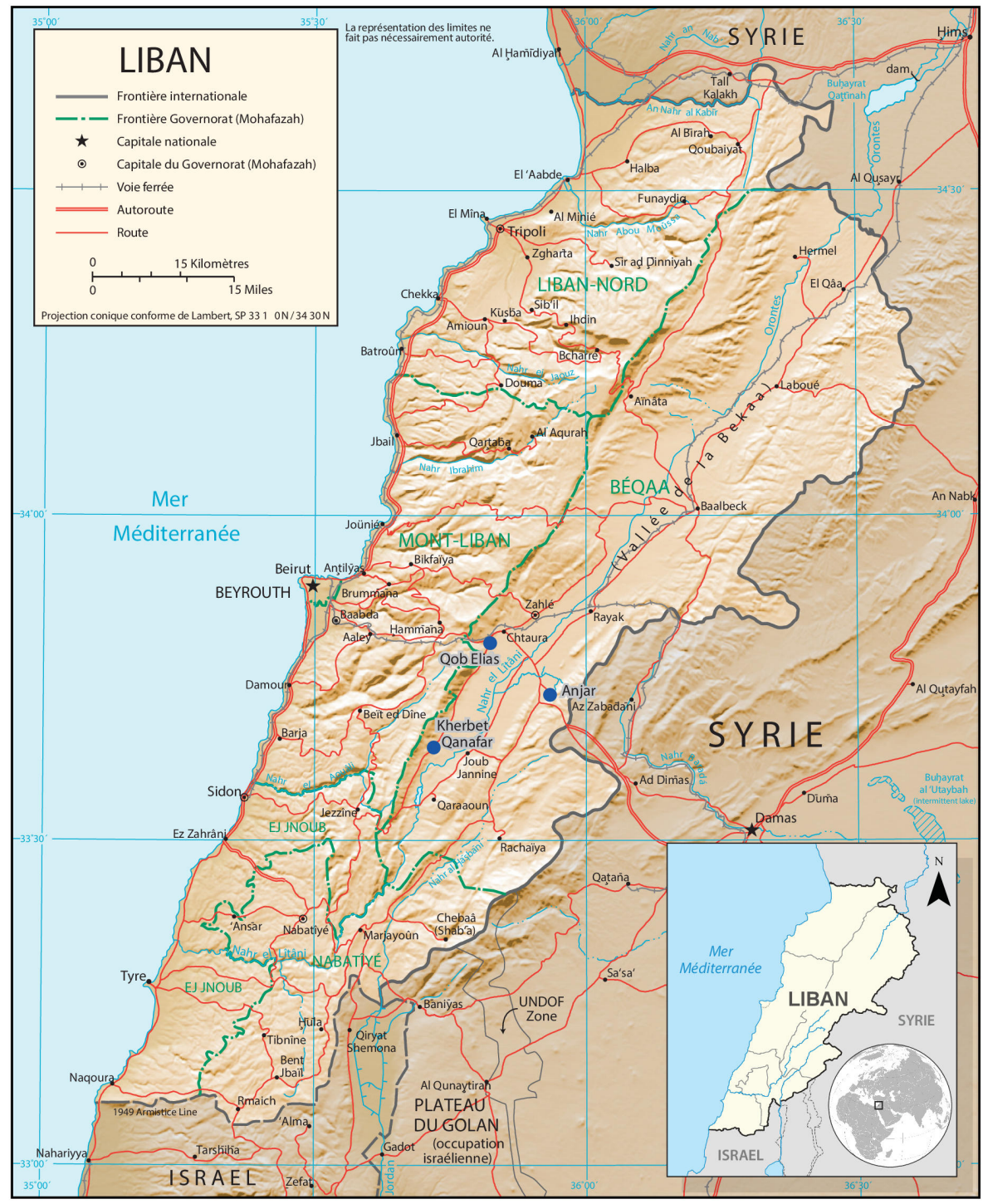

Modifiée par : Mourad Djaballah, technicien en cartographie, Département de géographie, UQAM Carte origianle produite par la US Central Intelligence Agency

Figure 1. Localisation des trois terrains d'études : Qoubb Elias, Kherbet Qanafar et Anjar. 


\section{Les terrains d'étude : Qoubb Elias, Kherbet Qanafar et Anjar}

Le village de Qoubb Elias se situe dans le district de Zahlé, à $15 \mathrm{~km}$ de Zahlé et à $45 \mathrm{~km}$ de la capitale libanaise Beyrouth. Son altitude moyenne est de $950 \mathrm{~m}$ et il s'étend sur $32 \mathrm{~km}^{2}$. la source du village «Ain » Qoubb Elias peut irriguer 6000 dunums $^{3}$. Cependant, 2500 dunums demeurent secs, car l'eau n'atteint plus la moitié aval du chevelu d'irrigation en gravitaire. L'exploitation qui a servi de premier cas d'étude se situe là. Son eau provient entièrement des puits artésiens et non pas de la source. Mais l'exploitation constituant le $3^{\mathrm{e}}$ cas d'étude dépend de la source de Qoubb Elias.
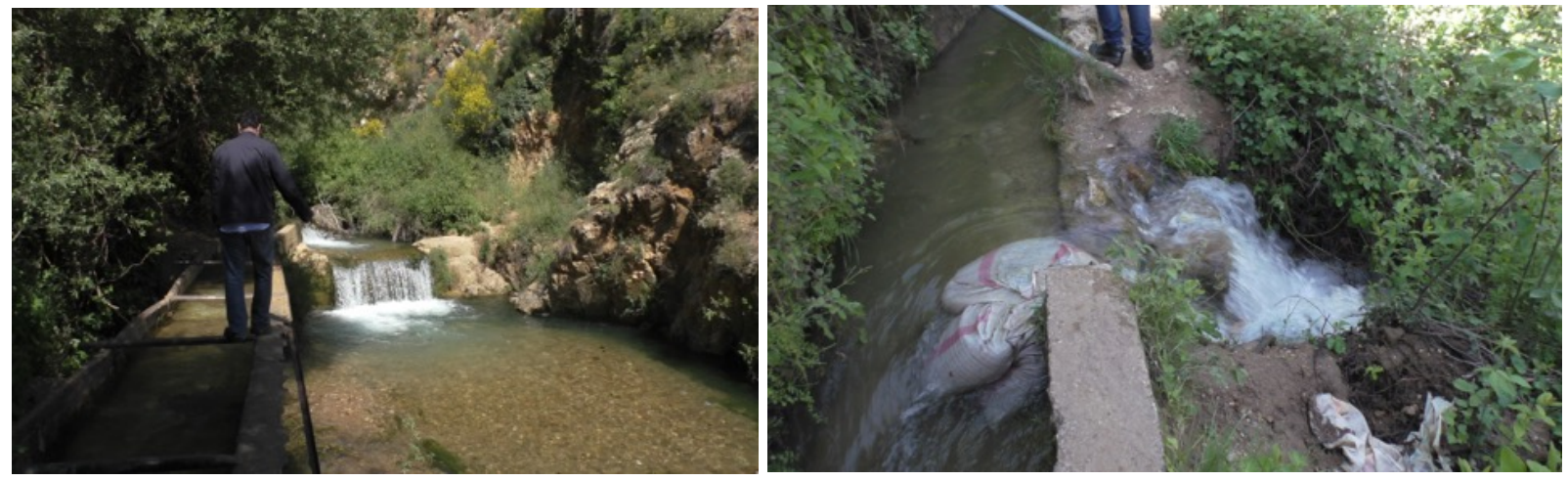

Figure 2:a) La source de Qoubb Elias. Les deux embranchements sont séparés par un muret. B) Des sacs de sable sur des bouts brisés du muret altèrent la répartition de l'eau entre les canaux de distribution.

Divers embranchements répartissent l'eau de la source de Ain Qoubb Elias tout au long de son parcours. Un système de tours d'eau qui reviennent tous les 20 ou 25 jours distribue le débit de la source au travers d'un chevelu de canaux vers les lopins des ayants droit. Le premier embranchement, situé en avant-plan de la figure 2, dirige l'eau vers 700 dunums où sont surtout cultivés des légumes ainsi que quelques arbres fruitiers. Ceci constitue un peu moins de $20 \%$ de la terre irriguée. La deuxième branche est elle-même divisée en deux. La première sous-division irrigue 850 dunums de terres où sont cultivés des arbres fruitiers et la deuxième, 1900 dunums ( $55 \%$ des terres irriguées) dont 1200 où sont cultivés des arbres fruitiers et 700 où sont cultivés des légumes. Le système de division et de répartition des embranchements, de l'amont vers l'aval des terres se concrétise par des ouvertures dans les parois des canaux de béton lorsque l'embranchement est proche de la source et que l'eau coule en permanence dans les deux branches, et par des petites digues ou portails (souvent métalliques) plus en aval, lorsque l'eau coule alternativement dans l'un ou dans l'autre des embranchements. Au total, le chevelu se compose de $34 \mathrm{~km}$ de canaux dont $14 \mathrm{~km}$ sont cimentés et $20 \mathrm{~km}$ en terre.

Un surveillant, désigné ici comme « chawwa » (نوّى) ou « natour 》 (ناطور), bloque et débloque les bifurcations des canaux en fonction de l'heure des tours d'eaux des uns et des autres. Les régimes de propriété communautaire, qui se construisent simultanément avec les chevelus d'irrigation à partir de sources, partagent systématiquement l'eau sur la base de tours mesurés en termes de temps. La durée du tour d'eau d'un ayant droit détermine la superficie

\footnotetext{
${ }^{3}$ Le dunum est la mesure de la terre agricole la plus utilisée par les agriculteurs du Liban et de certains autres pays du Levant. Il équivaut à 0,1 hectare.
} 
qu'il pourra irriguer. Elle est généralement fixée en fonction de l'apport originel à la construction du réseau d'irrigation apporté par le premier détenteur de ce droit. Lorsque sa terre est ensuite héritée ou vendue, ce droit d'eau lui reste lié. L'avantage d'un tel système, aussi inégalitaire soit-il, consiste à répartir de la même façon l'abondance en eau, lorsque le débit de la source est élevé, et la pénurie d'eau, lorsque le débit est faible. Comme le tour d'eau revient régulièrement tous les 20 jours, la fluctuation du débit des sources, caractéristique des régimes hydrologiques méditerranéens, est ainsi répartie d'une façon jugée équitable par les ayants droit.

Malgré l'abondance apparente de la ressource en eau, les terres situées à l'aval du réseau sont quelquefois laissées en jachère, faute d'eau pour les irriguer. La question du lien entre les puits et l'incapacité de la source à approvisionner en eau toutes les terres a été peu évoquée par les usagers. Cette question ne semblait pas causer de conflits sociaux sévères, mais elle préoccupait l'administration locale qui devait gérer un climat de discorde entre usagers désireux d'augmenter leur part de consommation. Selon le maire de Qoubb Elias, cette municipalité comptait, au moment de l'enquête, près de 43000 Libanais et 30000 Syriens. Plus de 35000 Libanais travaillent dans le secteur agricole. De nombreux Syriens arrivés au village après les conflits armés de 2011, avaient auparavant travaillé comme ouvriers agricoles saisonniers. Le retour de ces travailleurs détenant cette fois le statut de réfugiés a modifié les conditions socio-économiques liées au secteur agricole ainsi que l'accès à la ressource en eau.

Les réfugiés syriens présents au moment de l'enquête habitaient des camps de réfugiés localisés sur des terres louées. Leurs habitations de fortune, de simples tentes portant l'inscription de l'UNICEF ou du UNHRC se transforment petit à petit en structures de béton, signes d'un enracinement de cette population. La plupart, y compris les femmes, travaillaient comme ouvriers agricoles journaliers. Ils ouvraient aussi des petits commerces pour servir les autres réfugiés. Une pérennisation de leur présence s'esquisse.

La région de Kherbet Qanafar, beaucoup moins peuplée que Qoubb Elias, se trouve au sud de cette dernière (voir la Figure 1). Trois des exploitations visitées, toutes irriguées par l'eau de la source de Kherayzat (ain Kherayzat), se situent ici.

Le village de Anjar est situé dans le district de Zahlé, à $50 \mathrm{~km}$ de la capitale libanaise, Beyrouth. Il s'élève à $950 \mathrm{~m}$ d'altitude et s'étend sur $15.5 \mathrm{~km}^{2}$. Fondé à l'époque omeyyade, au début du $8^{\mathrm{e}}$ siècle, le village de Anjar est réputé pour les ruines de sa forteresse omeyyade. En 1939, la France contribue à l'installation des Arméniens originaires du territoire d'Alexandrette. Elle attribue 7 dunums à chaque habitant. Actuellement, le village compte plus de 2400 habitants, en majorité arméniens et propriétaires de leurs terres. La structure de la propriété formelle en lopins de 7 dunums persiste bien que certains propriétaires aient vendu leur bien à des habitants locaux (Figure 3a). Certains propriétaires cultivent leur lopin de terre eux-mêmes, mais la majorité les confie en location à d'autres agriculteurs arméniens du village, ou à des entrepreneurs qui les regroupent en grandes exploitations. La gestion de la distribution de l'eau constitue l'activité principale d'une association regroupant les agriculteurs de Anjar. 


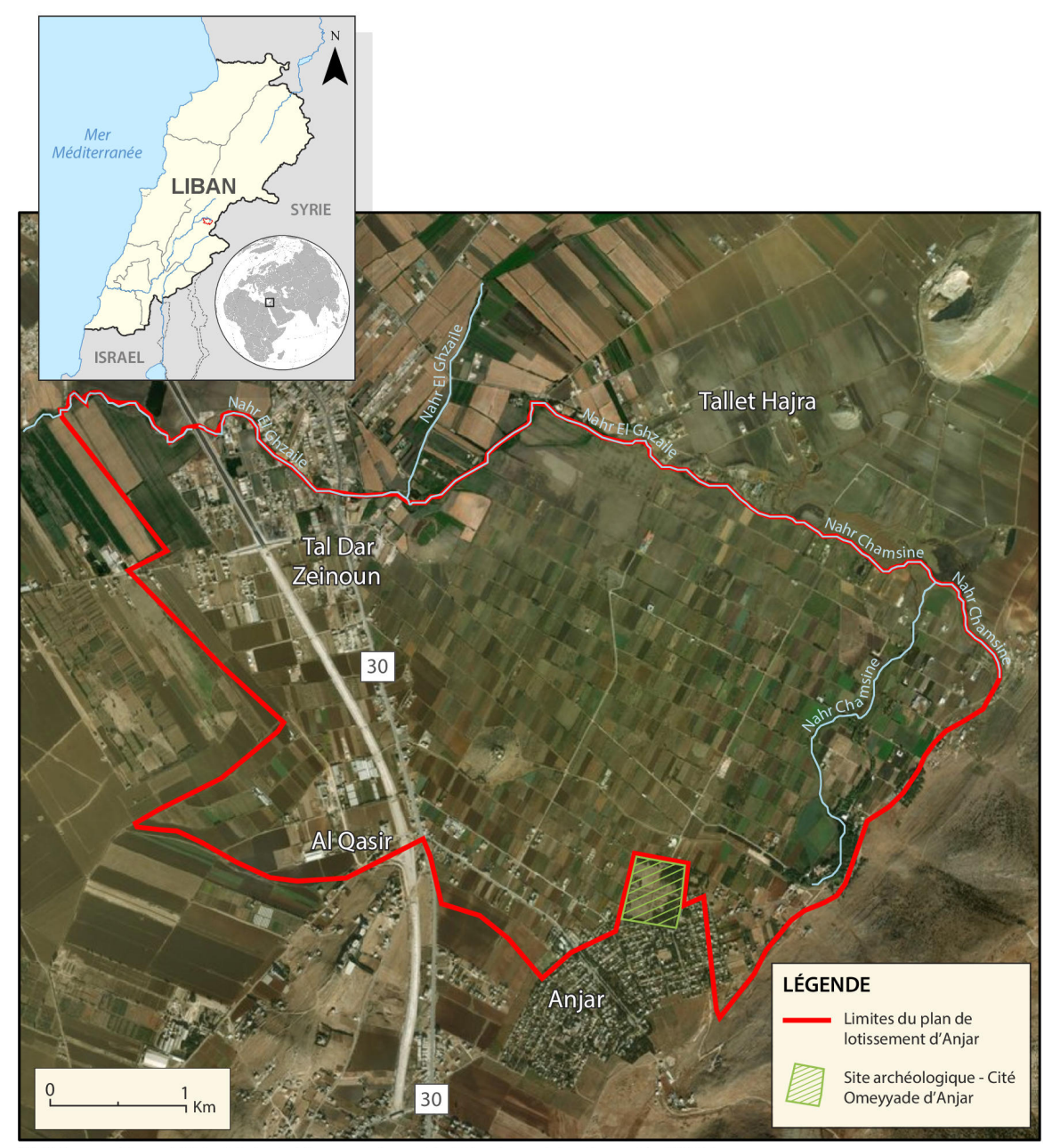

Réalisée par : Mourad Djaballah, technicien en cartographie, Département de géographie, UQAM

Image satellite: Esri, DigitalGlobe, GeoEye, Earthstar Geographics, CNES/Airbus DS, USDA, USGS, AeroGRID, IGN, and the GIS User Community 
Figure 3 : a) Vue satellitaire des plans de lotissement des terres de Anjar, attribuées à la communauté arménienne. On voit la subdivision de ces terres en lots de 7 dunums mais une partie de ces terre a été urbanisée.

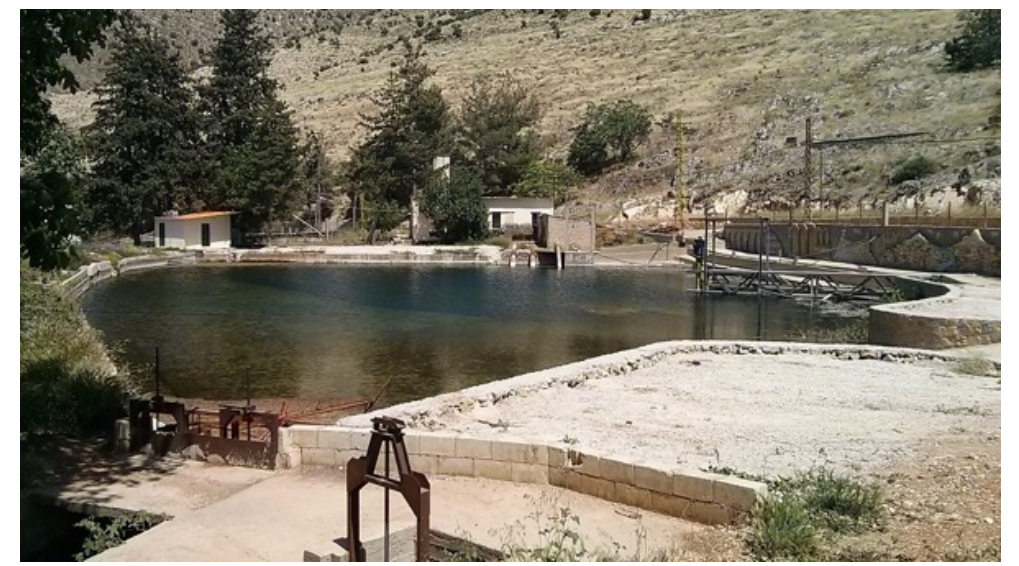

Figure $3: b$ ) les ouvrages de béton servant à contrôler l'eau de la source de Anjar.

Toutes les terres agricoles de Anjar sont irriguées par la source du même nom (Figure 3b), qui se divise en deux cours d'eau principaux : Chamsine et Ghozeil, avant l'acheminement de l'eau via des canalisations ouvertes ou cimentées. Ces rivières se jettent ensuite dans le Litani. Le débit de source est de $7200 \mathrm{~m}^{3} / \mathrm{h}$ en moyenne annuelle $\left(9432 \mathrm{~m}^{3} / \mathrm{h}\right.$ en hiver et $5148 \mathrm{~m}^{3} / \mathrm{h}$ en été).

L'eau de la source peut être acheminée par gravité à l'ensemble de la surface agricole de Anjar, mais des pompes sont quelquefois utilisées pour amener l'eau des rigoles vers les champs, et quelquefois pour remplir les rigoles d'eau quand la rivière est à débit d'étiage. Des digues aménagées sont contrôlées, afin de diriger l'eau dans un canal ou un autre. Près de la source Anjar, deux puits artésiens profonds ont été creusés jusqu'à la nappe souterraine afin de compenser la baisse du débit constatée notamment lors de la saison 2013/2014, période de précipitations très basses. Ces puits produisent $300 \mathrm{~m}^{3} / \mathrm{h}$ et leur eau est acheminée par des canalisations vers les terres agricoles.

\section{Les différentes modalités opératoires du lien tenure foncière - tenure de l'eau}

Notre recherche a permis d'identifier quatre cas de figure dans la co-construction de la tenure de la terre et de l'eau:

a -Les grands propriétaires qui gèrent eux-mêmes leur terre (et leur eau) - exemple de Monsieur A.

b - Les propriétaires absents, cas illustré par les terres de la famille $\mathrm{F}$.

c - Les petits propriétaires qui combinent propriété et location de terrains, cas illustré par M. M et par M. W,

d - La gestion communautaire de l'eau, situation illustrée par la communauté arménienne du village de Anjar.

\subsection{Les propriétaires gérant directement leur foncier}


La transformation de la tenure foncière à Qoubb Elias et son lien avec l'évolution de la tenure de l'eau se manifestent à travers le parcours de plusieurs propriétaires. Monsieur " $\mathrm{A}$ » fournit un cas typique. Ayant hérité de 30 dunums à la fin des années 1960, le propriétaire devint prospère grâce à son activité agricole, mais aussi commerciale. Tout au long de son parcours d'homme d'affaires et d'agriculteur avisé, il entreprit l'achat de lopins de terre agricole, jusqu'à cumuler 500 dunums constitués de lopins pas toujours contigus. La famille devint influente localement, et son frère accéda au poste de maire de la municipalité, une position de prestige qui permettait sans doute d'augmenter le poids économique de la famille.

Cette progression foncière est ralentie après l'éclatement de la bulle financière. Plusieurs détenteurs de capitaux libanais investissent dans l'achat de terres agricoles à partir de 2009, contribuant ainsi à la hausse des prix du foncier. Face à cette situation, le propriétaire a pu persister dans le système foncier en diversifiant ses activités commerciales, mais surtout en continuant à développer de façon systématique et rationnelle la gestion de sa production agricole et de l'eau nécessaire. La totalité de ses 500 dunums, qui requièrent une consommation conséquente en eau, sont irrigués par un puits foré en 1979 dans la zone de Haql al-Jabal, près de Qoubb Elias. Monsieur « $A$ » développa lui-même un réseau de canalisations souterraines pour alimenter un système de goutte-à-goutte sur ses terres. Cette stratégie d'utilisation efficace de l'eau des puits lui permit de contourner l'ancien système de tenure de l'eau et de ne pas être limité dans son choix d'assolement par les pénuries potentielles. Parallèlement, il a expérimenté de nouvelles variétés d'arbres fruitiers importés (surtout des nectariniers), effectuant aussi des greffes de nouvelles variétés sur des souches existantes. Il tenait une comptabilité détaillée de la productivité de chaque variété et de ses besoins en eau.

Le propriétaire « $\mathrm{A}$ » avait pu acheter des terres cultivées auparavant en pluvial, à un prix inférieur à celui des terres irriguées par la source. La productivité des terres est assurée par le raccord de leur système d'irrigation au puits de $100 \mathrm{~m}$ de profondeur qui atteint la nappe phréatique. Un deuxième puits moins profond et dont le débit est plus faible est aussi utilisé en appoint. Très clairement, de tels puits ne peuvent qu'interférer avec le débit de la source, ce qui explique sans doute la faible disponibilité de l'eau d'irrigation provenant des sources avoisinantes.

Il s'agit ici typiquement d'un front pionnier interstitiel induit par un nouvel accès à l'eau. (Trottier et Perrier, 2018) L'agriculture s'intensifie soudainement, grâce à l'irrigation, sur une parcelle nichée entre des zones habitées et d'autres zones cultivées. L'assolement est changé. Le blé autrefois cultivé en pluvial est remplacé par des arbres fruitiers ou des légumes, récoltes plus rentables rendues possibles par ce système d'irrigation. L'ancienne tenure de l'eau, qui réglemente l'appropriation de l'eau de la source, correspond à un régime de propriété communautaire. La nouvelle tenure de l'eau développée par le propriétaire « $\mathrm{A}$ » et par la plupart des grands propriétaires fonciers correspond à une capture non réglementée de la ressource en eau. Contrairement au propriétaire " $\mathrm{A}$ », d'autres propriétaires de la localité n'habitent pas le village. Par conséquent, ce phénomène de concentration foncière s'est accompagné d'un effritement du capital social local parce que la gestion socio-économique de la ressource en eau repose sur une capture non règlementée 
de celle-ci par opposition à une gestion communautaire. Cette capture profite à une minorité disposant d'avantages politiques et économiques.

L'accès du propriétaire à l'eau dépasse largement ses besoins en irrigation. C'est le cas de plusieurs autres grands propriétaires fonciers. Cette situation aboutit à un accès inégalitaire à la ressource, qui produit des avantages tangibles au niveau individuel : la disponibilité de l'eau se traduira par une amélioration de la qualité des produits agricoles et permettra la protection des vergers de nectariniers par un système d'aspersion légère lors des périodes de gel tardif. Cette consommation d'eau est donc liée à la mise en valeur agricole, mais n'est pas comptabilisée dans le calcul de l'eau virtuelle contenue dans ces nectarines. Cette pratique découle de conditions climatiques spécifiques, puisque les vergers se situent presqu'à 900 mètres au-dessus du niveau de la mer et subissent des nuits froides au printemps. Mais elle découle aussi de facteurs sociaux liés à la tenure de l'eau. Si Monsieur A avait dû partager l'eau de la source avec ses voisins, il n'aurait peut-être pas eu accès à cette ressource additionnelle destinée à protéger les nectariniers du froid.

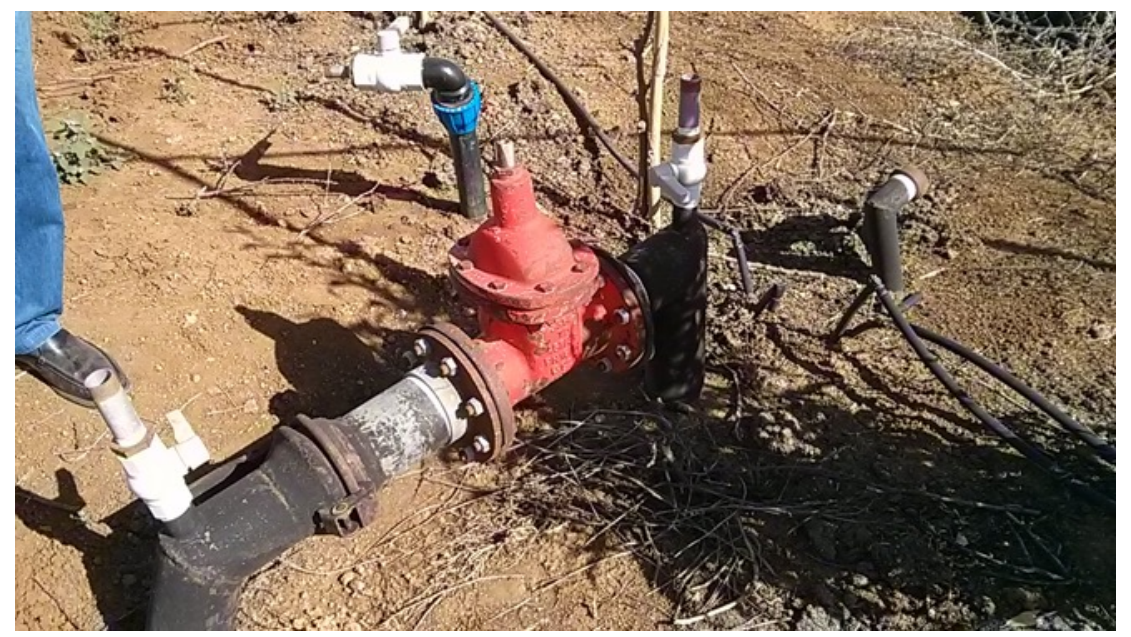

Figure 4 : Premier cas de figure: les propriétaires gèrent directement leur foncier. Des valves incorporées dans le réseau élaboré de tuyaux souterrains permettent une gestion optimale de l'eau, dont le débit est modifié en fonction des besoins variables des diverses variétés d'arbres fruitiers et de la température.

Une première concentration foncière a été réalisée au bénéfice de quelques propriétaires locaux à Qoubb Elias entre 1960 et 2009, grâce au nouveau type d'accès à l'eau que constituait le puits. Deux formes de tenure de l'eau co-existaient alors : la première est communautaire, qui gère la source et la seconde est une simple capture de l'eau non réglementée via le forage de puits. Cette première phase de transformation s'est interrompue dès 2009, lorsque plusieurs investisseurs libanais étrangers au village se sont intéressés au foncier. Le placement était jugé plus rentable face aux risques encourus sur les marchés financiers après la crise internationale de 2008. La hausse soudaine du prix du foncier qui résulta empêcha les propriétaires locaux de poursuivre leurs achats de terre. Les nouveaux investisseurs n'habitent pas la localité et confient la gestion de leurs biens à des gestionnaires locaux qui embauchent des travailleurs saisonniers. Même avant la crise syrienne de 2011, des travailleurs agricoles syriens migraient vers la Beqaa de façon saisonnière, année après année, et certains d'entre eux y résidaient de façon presque permanente. Mais l'arrivée massive de réfugiés syriens à partir de 2011 a permis d'alimenter 
ce nouveau système d'acquisition et de gestion de propriétés foncières. Cette main-d'oeuvre, sur place toute l'année, pourrait bien préférer des modes de métayage à l'avenir, un statut qui accorde plus de sécurité et d'indépendance que le travail d'ouvrier journalier. Actuellement, certains gestionnaires mettent à disposition des travailleurs syriens des logements modestes et équipés. L'enracinement des réfugiés syriens sur les terres agricoles de la Beqaa augure une nouvelle transformation foncière. D'autant que les gestionnaires offrent des salaires bien inférieurs à ceux acceptés par les ouvriers libanais. L'aide financière complémentaire accordée par l'UNHRC aux réfugiés favorise ce différentiel de rémunération.

On trouve donc à Qoubb Elias un premier phénomène d'intensification de l'agriculture lié à un nouvel accès à l'eau. Il s'agit de fronts pionniers agricoles interstitiels du même type que ce qui a été observé en Cisjordanie, notamment dans la vallée d'El Far'a (Trottier et Perrier, 2018). Ce phénomène puise son origine dans un contournement de la tenure de l'eau ancienne, élaboré pour gérer l'eau de source, grâce au forage de nombreux puits à compter des années 50 et 60. L'accès déréglementé à l'eau va entraîner une transformation foncière en même temps qu'une disparition de la source et un affaiblissement de la tenure de l'eau communautaire qui la caractérisait. À partir de 2009-2010, une nouvelle transformation agricole s'esquisse, causée en partie par la crise financière internationale de 2008 qui encourage les investisseurs à acheter des terres agricoles et par le conflit syrien qui alimentera ensuite l'arrivée d'un nombre important de réfugiés dans la région à partir de 2011. Ce second phénomène peut se comparer à la transformation agricole de la vallée du Jourdain en Cisjordanie à partir de 1948, lorsqu'un afflux massif de réfugiés contribua à la transformation de la tenure foncière.

\subsection{La tenure foncière par des propriétaires absents}

Un second type de gestion foncière s'est développé à partir des années 50 dans la Beqaa : la gestion agricole au nom de propriétaires absents. Voici le cas d'une famille « $\mathrm{F}$ » de commerçants de Beyrouth qui possède 120 dunums dans le village de Kherbet Qanafar, à proximité de Qoubb Elias. Cette terre est gérée depuis quatre ou cinq ans par un agronome résidant à Beyrouth qui possède lui-même des terres agricoles au nord de la Beqaa. Ce gestionnaire s'y rend chaque samedi pour examiner les produits agricoles, s'assurer du bon fonctionnement du système d'irrigation et payer les ouvriers, tout en leur assignant les tâches à exécuter au cours de la semaine suivante.

Les terres de la famille « $\mathrm{F} »$ sont surtout plantées de vignes dont les produits sont commercialisés, mais également d'arbres fruitiers divers (cerisiers, pommiers, pêchers, cognassiers) pour une consommation familiale. Un petit lopin de 5 dunums est réservé à une nouvelle plantation de jeunes pommiers pas encore productifs, un élevage de volailles et un potager, le tout entretenu par un jeune ouvrier agricole syrien qui vit en permanence sur place. Des discussions concernant l'usage de la terre resurgissent à l'occasion entre le gestionnaire agronome et l'ouvrier agricole, notamment à propos des tâches assignées par le gestionnaire, qui doivent se concilier avec les initiatives prises par l'ouvrier. Un autre ouvrier agricole plus âgé, Libanais et résident du village, lui-même petit propriétaire, supervise le premier et s'occupe également de la gestion quotidienne des parcelles et de l'embauche des journaliers lorsque nécessaire. Visiblement, les deux ouvriers bénéficient de 
la confiance de la famille propriétaire ainsi que de celle de l'agronome-gestionnaire pour la gestion quotidienne, mais c'est ce dernier qui prend les décisions importantes.

La modalité de gestion actuelle est l'aboutissement d'une longue histoire. La famille en question travaillait dans le secteur commercial à Beyrouth et a acheté des terres dans la plaine de la Beqaa à partir des années 1950. Elle a ensuite agrandi son domaine graduellement. Cet investissement foncier a été hérité par les enfants de la famille, mais ces derniers s'intéressent peu à l'agriculture. La gestion technique et commerciale de l'ensemble des terres de la famille « $\mathrm{F}$ » a donc été déléguée à un agronome qui a réussi à améliorer grandement les rendements et à aboutir à une plus-value foncière importante.

En effet, l'agronome a amélioré le rendement des vignes et a lancé un appel d'offres public pour l'achat des récoltes de raisins, ce qui a permis de quadrupler les revenus et de pérenniser un marché sous contrat d'exploitation et de commercialisation des raisins sur 10 ans. Il faut dire que la région est connue pour ses vins et son arak de qualité supérieure. L'effet combiné de l'amélioration de la production et des conditions de vente ont permis de basculer vers une nouvelle forme d'exploitation plus marchande.

Le cas de la famille propriétaire « $\mathrm{F}$ » illustre ainsi une transformation lente et certaine. La gestion foncière paternaliste exercée par un propriétaire absent, pour qui maintenir des liens de confiance avec ses employés et ses acheteurs l'emportaient sur la rentabilité de l'exploitation, a été remplacée par une gestion néolibérale où la productivité et la rentabilité sont désormais les objectifs premiers. La sécurité du logement et les avantages dont le jeune ouvrier agricole bénéficie (culture de potager, élevage) ressemblent aux conditions dont jouissent souvent les métayers dans la région. L'employé plus âgé, qui est aussi un voisin et qui travaille pour la famille depuis des décennies, a bénéficié de la promesse du père (décédé) de ne jamais être renvoyé. Le gestionnaire agronome, lui, administre la propriété sur la base de relations contractuelles. La famille propriétaire n'entretient plus de relations paternalistes avec les ouvriers agricoles journaliers, qui deviennent une ressource substituable au gré des variations du marché.

L'exploitation est alimentée dans son ensemble par la source de Kherayzat, située en amont sur une hauteur, à la jonction de la plaine avec le flanc Est du Mont-Liban. L'eau parvient aux parcelles par effet de gravité, mais l'installation de pompes a permis son acheminement du canal jusqu'aux cultures. Un surveillant (natour) accorde les tours d'eau aux divers ayants droit. Les parcelles agricoles et l'exploitation de la famille propriétaire « F » se situent en amont du canal de distribution, ce qui assure une stabilité de l'approvisionnement en eau et permet l'installation de systèmes de goutte à goutte sur certaines parcelles.

La famille propriétaire, malgré son absence, a bénéficié de la proximité de sa terre avec la source, en amont du système d'irrigation actuel, grâce à l'achat initial d'une exploitation qui servait de maison de campagne, bien plus pour l'agrément que comme source de revenus. La taille de la propriété agricole et la proximité de la source ont permis d'orienter l'exploitation vers une production marchande, sans investir dans un puits.

\subsection{Les petits propriétaires fonciers}


Les petits propriétaires fournissent des exemples complexes de tenure de l'eau et de la terre, qui mêlent des contractualisations monétarisées et non monétarisées. Trois cas d'étude illustrent divers types de gestion du foncier et de la ressource en eau.

i) Le premier cas d'étude est celui de Monsieur «I» qui est à la fois employé et gardien de l'exploitation d'une famille de propriétaires absents. Il est lui-même propriétaire de 11 dunums, qu'il cultive en employant des ouvriers agricoles. L'ouvrier-propriétaire âgé de 70 ans environ gère sa terre située à $2 \mathrm{~km}$ de celle de la famille des propriétaires pour qui il travaille, mais en aval du chevelu d'irrigation. Ses parcelles sont irriguées elles aussi à partir de la source de Kherayzat, mais elles ne reçoivent plus suffisamment d'eau. Il compense le manque d'eau par l'achat d'eau qui vient d'un puits artésien voisin profond de 50 m.

M. «I » loue également une partie de sa terre à un autre agriculteur qui la cultive. Le lien de location permet des collaborations et échanges concernant l'eau. Dans ce cas, le locataire de $\mathrm{M}$. «I I, qui possède un puits artésien sur une autre terre dont il est propriétaire, fournit l'eau manquante à la terre de M. « I ». Il arrose les arbres de M. «I » tous les 20 jours, et ceci constitue le prix de la location : l'eau est fournie en échange de la terre louée. Le locataire cultive ses légumes entre les rangées de jeunes arbres que $\mathrm{M}$. «I » a plantés cette année. Dans deux ans, il ne faudra plus planter de légumes entre les arbres car ceux-ci seront devenus trop grands pour le tolérer sans perdre en productivité. Un nouvel arrangement devra être négocié.

ii) Monsieur « $\mathrm{M}$ » est détenteur d'une exploitation de 100 dunums à Kherbet Qanafar, dont 40 dunums hérités, 40 dunums achetés au cours des 20 dernières années et 20 dunums loués. Il travaille à temps plein comme propriétaireexploitant et gère directement ses parcelles. Dans les années 1960, cet agriculteur cultivait des légumes et n'avait que 15 dunums dédiés aux arbres fruitiers. Il diversifia sa production à partir de 1967 (nectarines, pommes, poires et cerises) pour des raisons de rentabilité. $\mathrm{M}$. « $\mathrm{M}$ » vend aux grossistes et exporte une partie de sa production vers le marché extérieur. Les parcelles agricoles de $M$. « $M$ » sont localisées à quelques kilomètres de la source de Kherayzat. Au début, il cultivait du blé et des légumes, en alternant les cultures chaque année. Depuis 1950, le forage de puits s'est intensifié dans la région, car l'approvisionnement en eau n'était plus suffisant pour alimenter les exploitations de plus en plus orientées vers l'arboriculture.

De 1950 à 1955, plusieurs agriculteurs de la localité se mettent à cultiver des légumes, mais les puits situés à Kherbet Qanafar ne fournissent pas assez d'eau aux nouvelles cultures. En 1966, le père de $M$. « $M$ » achète une terre adjacente à la sienne, approvisionnée par un puits artésien situé hors de cette terre, à

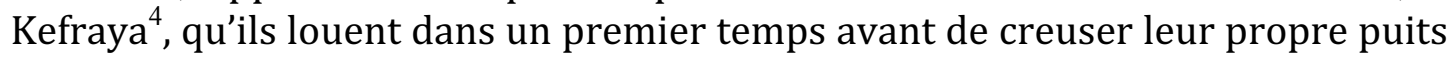
sur leur terre. Tous les agriculteurs du village et des villages voisins se mettent

\footnotetext{
${ }^{4}$ La terre de Monsieur M. se trouve dans Kherbet Qanafar, près du village de Kefraya.
} 
ainsi à creuser des puits. Plus de 50 puits existent aujourd'hui dans le village. Pendant les périodes de faibles précipitations, les agriculteurs se voient contraints de les approfondir. Les propriétaires de puits au débit faible doivent alors acheter de l'eau provenant de puits plus profonds. La pénurie générale d'eau a incité les agriculteurs à installer des systèmes de goutte-à-goutte pour rationaliser la consommation, sans pour autant aboutir à une solution pérenne d'exploitation de l'eau.

iii) Le troisième cas d'étude est celui d'un employé de la municipalité de Qoubb Elias qui est locataire de terres agricoles. Monsieur « $W$ » loue 130 dunums à prix fixe afin de les exploiter de la façon suivante : 60 dunums pour la culture du blé, 40 dunums d'arboriculture, 25 dunums pour la culture de pois, et 5 dunums pour des cultures sous serres. Le blé est cultivé pendant l'hiver, et demande parfois un arrosage additionnel à la fin du printemps. Les arbres fruitiers sont arrosés tous les 25 jours grâce à la source de Qoubb Elias. L'eau suffit en général, mais à partir du mois de juillet, l'arrosage est moins régulier et plus espacé dans le temps en raison du stress hydrique. Ce locataire de parcelles dépend entièrement de la source de Qoubb Elias. Il accumule l'eau prélevée durant son tour d'eau dans un réservoir qu'il a creusé. Il a ainsi pu passer d'une gestion de flux à une gestion de stock d'eau.

Ces trois cas d'études de petits exploitants montrent ainsi une grande variété de stratégies d'adaptation. Depuis les années 1950, ils ont progressivement délaissé la culture du blé au profit de cultures plus rentables, mais plus consommatrices d'eau. Ils ont intégré des pratiques marchandes, allant jusqu'à l'exportation ciblée de leurs récoltes, avec des pratiques non marchandes de bon voisinage pour sécuriser leur accès à l'eau et à la terre. Ils pratiquent aussi bien la polyculture que la monoculture en fonction des circonstances et des arrangements entre agriculteurs et propriétaires. Leurs besoins croissants en eau les a menés à multiplier les forages de puits et, par ricochet, à diminuer les débits des sources. Pourtant, ce sont souvent ceux-là mêmes qui bénéficiaient des sources. Aucune institution publique ne semble, jusqu'à présent, être intervenue pour initier un débat entre usagers afin d'aboutir collectivement à un plan de gestion de ces deux types d'approvisionnement en eau (puits et sources) afin de pérenniser l'exploitation de la ressource. Les agriculteurs poursuivent ainsi inexorablement une course à la ressource qui entraînera, à terme, le tarissement de la nappe phréatique. La situation est assez similaire à celle de la vallée d'El Fara, en Cisjordanie, où les puits se sont multipliés à partir de 1994, aboutissant déjà à un tarissement des sources (Trottier and Perrier, 2018).

\subsection{La gestion communautaire de l'eau dans le village de Anjar}

Dans le village de Anjar, l'exploitation agricole est essentiellement communautaire. Prenons le cas de Monsieur « $K$ », à la fois agriculteur et entrepreneur, qui gère sa propre parcelle de 7 dunums. Ces lopins lui ont été octroyés au départ comme à tous les réfugiés arméniens installés à Anjar. L'agriculteur-entrepreneur gère également d'autres parcelles qu'il a achetées, en loue d'autres et dispose de contrats pour irriguer les parcelles d'autres agriculteurs. Au total, il possède 40 dunums, loue près de 100 dunums et irrigue 50 dunums 
qui ne lui appartiennent pas, contre rémunération. Il irrigue 140 dunums à partir de la source Anjar, via l'une ou l'autre des deux rivières (Chamsin et Ghozeil). Les 50 dunums restants sont irrigués avec de l'eau provenant de puits artésiens.

M. « K » possède des arbres fruitiers (pommiers, pêchers et nectariniers) et plante des légumes (courges). La production de pommes atteint généralement 40 tonnes par an. En cas d'aléa climatique, comme lors de l'épisode de froid extrême de 2014, la production de pommes n'a pas dépassé $100 \mathrm{~kg}$. La même année, un autre aléa, de nature politique, a pesé : la route de l'exportation vers les pays du golfe arabo-persique, qui passe par la Syrie, n'étant plus accessible, il a fallu entreposer les produits dans un entrepôt réfrigéré. Le coût de la location (5000 \$) a dépassé les recettes (4500\$).

L'agriculteur-entrepreneur utilisait auparavant un système de goutte à goutte car l'eau était limpide. Cependant, la rivière Ghozeil est de plus en plus turbide, ce qui conduit à l'obstruction des buses de distribution par des sédiments. Le système d'irrigation par immersion dans des sillons, qui consomme plus d'eau, a donc été utilisé à nouveau.

En 2014, le débit de la source de Anjar tarit à cause de la faible recharge de la nappe. Ce tarissement avait été signalé dès 2010. Depuis, les agriculteurs font face à plusieurs problèmes d'approvisionnement en eau et doivent s'adapter à la pénurie, par exemple par la pose de pompes mobiles sur la rivière Ghozeil. C'est le canal de distribution principal qui est réalimenté en eau à partir de la rivière pour desservir les terres. Une organisation collective entre agriculteurs permet de faire fonctionner des pompes fixes non motorisées à l'aide d'un tracteur connecté (Figure 5). Ces actions collectives sont coordonnées par une organisation, «L'association de l'eau de Anjar ». Le centre de l'association est aussi un lieu de rencontre et de socialisation pour les agriculteurs. Au moment de la visite de terrain, un petit canal bétonné était en réfection. C'est l'association qui supervisait le travail et plusieurs agriculteurs suivaient l'évolution des travaux.

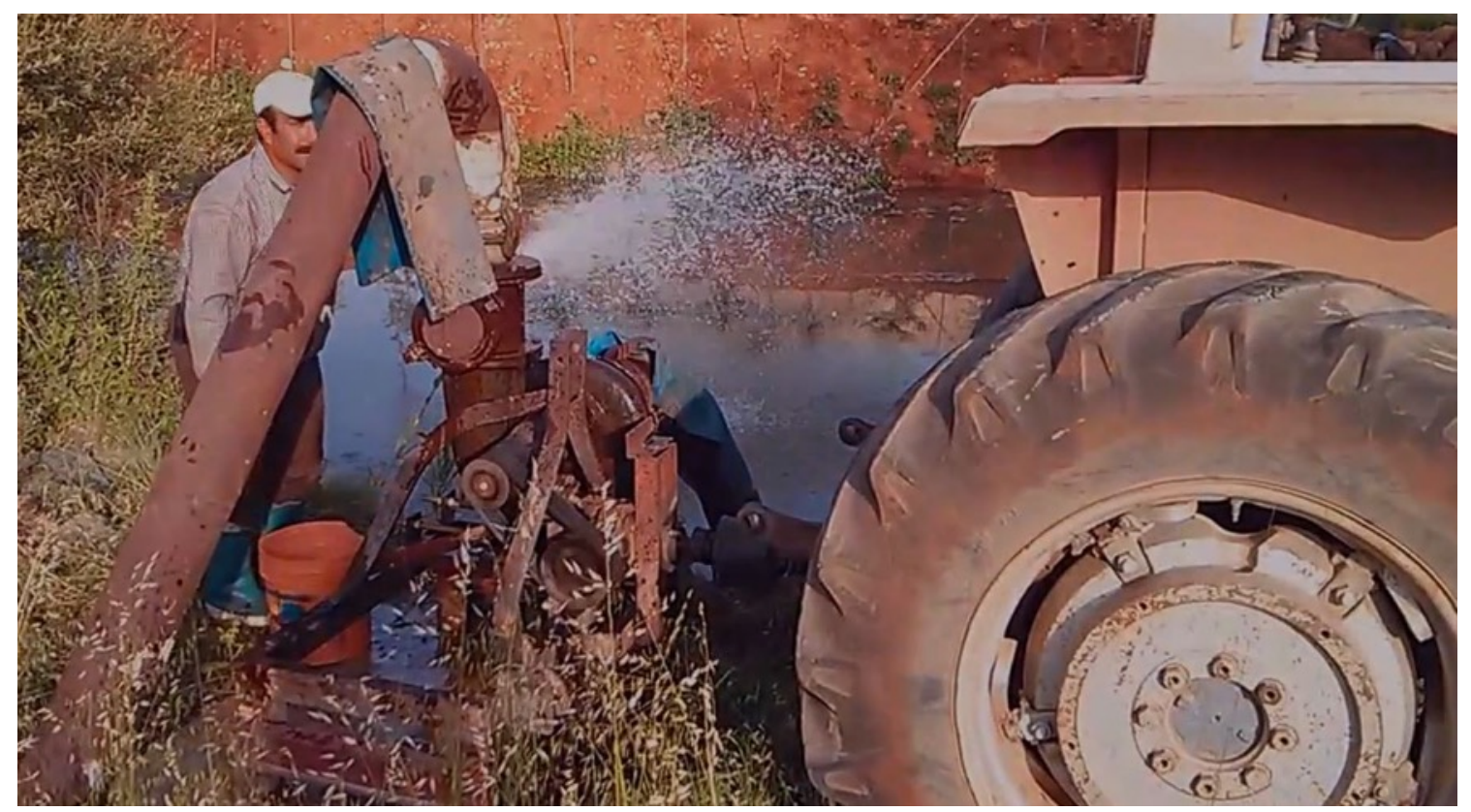


Figure 5 : Un tracteur est connecté à une pompe fixe non motorisée pour alimenter un canal à partir de la rivière Ghozeil.

\section{La critical political ecology et la co-production de la tenure de la terre et de l'eau}

Cette enquête est essentiellement qualitative et ne vise pas à établir des rapports de causalité statistique entre les caractéristiques des exploitations et les modalités d'accès à l'eau. Cependant, des logiques d'action sont identifiées et interprétées à la lumière de la théorie de la critical political ecology. Ceci permet de mettre en évidence l'influence des facteurs sociaux et politiques qui structurent le rapport des acteurs aux ressources naturelles et les options d'adaptation qui s'offrent à eux. Plusieurs observations se dégagent des entrevues qui mériteraient d'être confirmées par des recherches futures.

Le rapport diversifié à la ressource en eau, en fonction de la taille des exploitations et de la position sociale de leurs propriétaires, n'est pas strictement corrélé aux conditions géographiques, mais plutôt aux conditions sociales et politiques. Ce sont les facteurs économiques et géostratégiques qui amènent les agriculteurs à modifier leur production agricole, ainsi que les modalités de leur accès à l'eau et à la terre.

Quant aux conditions sociales qui régissent le rapport des acteurs aux ressources naturelles (eau et terre), on constate une différence nette, par exemple, entre les solutions d'adaptation adoptées par les agriculteurs riches et les moins fortunés. Les premiers ont tendance à se procurer des pompes, plus ou moins puissantes selon la surface à irriguer, même quand les terres agricoles sont localisées le long du canal d'irrigation. Cet avantage socio-économique leur procure plus d'indépendance vis-à-vis des structures décisionnelles collectives et plus de sécurité face aux fluctuations de la température et de la quantité d'eau. Par contre, les agriculteurs moins nantis sont dépendants du système du tour d'eau. Même si ce système de gestion n'assure pas la totalité des besoins pour l'irrigation (apport supplémentaire moyennant des frais), le procédé de répartition permet d'éviter des crises. Il est clair que le droit d'accès à l'eau est lié à la terre, que l'on soit locataire ou propriétaire. Cependant, les modalités d'accès et d'utilisation de la ressource hydrique est affecté par des conditions socio-économiques.

Les structures décisionnelles locales (municipalité ou association d'usagers) entretiennent les infrastructures de distribution. Cependant, leur capacité de développement des infrastructures est limitée. Les agriculteurs riches, par exemple, ont les moyens financiers et politiques de transformer leurs infrastructures et les adapter aux changements du marché, entraînant ainsi des changements dans les types de cultures adoptées. Lorsque les mutations découlent des fluctuations du marché international, des opportunités et coûts d'exportation, les agriculteurs nantis s'ajustent plus facilement en modifiant les types de cultures.

Dans la plaine de la Beqaa, les stratégies d'adaptation aux conditions économiques et géostratégiques changeantes ont consisté à passer d'une culture à une autre (arbres fruitiers), et à adopter un système de pompage et d'arrosage au goutte-à-goutte, plutôt que de transformer profondément l'infrastructure d'irrigation au niveau local. Ces actions 
découlent de l'absence : i) d'une stratégie nationale de gestion et de distribution de l'eau, ii) d'aide aux agriculteurs et iii) d'investissements dans les infrastructures.

Etant donné que la tenure de l'eau n'est pas institutionnalisée et balisée par des règles comme c'est le cas pour la tenure de la terre, cela permet aux agriculteurs nantis de mieux se prévaloir de la ressource en eau. Si cette situation entraîne des inégalités et affecte l'efficacité d'ensemble du système, elle ne semble pas provoquer de conflits importants. Par exemple, les terres situées à l'amont d'un canal en terre reçoivent plus d'eau. Disposant de suffisamment d'eau, les propriétaires de ces terres ne cimentent pas les canaux, n'installent pas de tuyaux et n'utilisent pas de membranes géotextiles pour étanchéifier les canaux en terre. Ainsi, un volume d'eau important s'infiltre dans le sol. Une autre conséquence claire de ces déperditions par infiltration au travers des canaux en terre est la recharge des puits, situés en contrebas de la source. Les usagers situés à l'extrémité aval des canaux sont désavantagés. Cependant la situation n'est pas assez critique pour provoquer des conflits, compte tenu du volume d'eau disponible chaque année.

Si l'on compare les modalités de gestion de la ressource en eau dans la Béqaa avec celles utilisées dans le Delta du Nil (Égypte), on constate un mode de fonctionnement différent. La question de l'eau étant critique dans le Delta du Nil, compte tenu de la faible disponibilité hydrique, les communautés villageoises ont développé des mécanismes de résolution des conflits fondés sur les rapports sociaux communautaires. Par exemple, il n'est pas rare que des paysans détenteurs de petits lopins situés en bout de rigole d'irrigation contractent des alliances familiales avec ceux qui possèdent des lopins au début de la rigole (Antonius, 1992). Cela permet de traiter l'accès différentiel à la ressource comme un problème interne à la famille, ce qui assure un règlement plus facile des conflits.

D'autres facteurs influencent la distribution de la ressource en eau. La tenure foncière est nécessairement coproduite en interaction avec la représentation de l'espace. (Le Roy, 2011) Le Roy reprend plusieurs types de représentation de l'espace issues de la chorématique française. Cet article n'en reprend que deux: la représentation géométrique et la représentation topocentrique. Au sein d'une représentation géométrique, l'espace est mesuré en fonction de coordonnées et peut être cartographié dans son ensemble. Il s'agit de la représentation sur laquelle se base un cadastre, par exemple. Au sein d'une représentation topocentrique, l'espace est lié à un point particulier et organisé autour de ce point. Ce point peut être le site d'une fonction politique, économique ou religieuse. L'exploitation des sources au travers de chevelus d'irrigation se construit sur base d'une représentation topocentrique de l'espace. Tout y est organisé par rapport à la source puisque l'eau doit être acheminée par gravité. En revanche, l'exploitation par puits suppose une représentation géométrique de l'espace. Ce dernier mode d'organisation est avantageux pour les investisseurs non-locaux qui se dispensent des liens de bon voisinage nécessaires pour assurer une interdépendance fructueuse en cas de pénurie.

Or, la trajectoire spatiale de l'eau dans les chevelus d'irrigation est affectée par l'imperméabilisation ou non des canaux, soumettant ainsi les conditions d'usage de l'eau à des règles sociales. Les propriétaires des puits bénéficient et maintiennent le système des canaux en terre, permettant ainsi une plus grande infiltration souterraine qui alimente ces 
puits. Cette trajectoire spatiale de l'eau s'accompagne d'une trajectoire plus institutionnalisée quand l'eau circule dans le canal d'irrigation. La ressource en eau est ainsi gérée selon les règles établies par le régime de propriété communautaire de la source. Au contraire, lorsque l'eau est interceptée par le puits, la ressource est appropriée par simple capture, totalement déréglementée.

\section{Conclusion}

Les cas d'étude de cette enquête illustrent différentes manières d'appréhender les modalités de co-production de la tenure de l'eau et de la tenure foncière dans la Beqaa (Liban). Dans le village de Anjar, l'organisation du foncier et de l'irrigation est spécifique par rapport aux autres villages de la Beqaa. Les différentes communautés villageoises disposent de leur propre définition de ce qui constitue une gestion légitime et efficiente de la terre et de l'eau. Des tenures foncières et des tenures de l'eau très différentes se sont co-produites, même dans le cadre d'un écosystème ou d'une topographie aux caractéristiques similaires, d'un seul système national et sous un même climat. Les cas étudiés ici permettent de comprendre comment, dans chaque cas, les ressources de l'eau et de la terre sont gérées.

Les constats précédents confortent et confirment une orientation théorique, celle de la critical political ecology. Nous avons vu en effet que la construction d'un discours sur l'environnement n'est jamais indépendante des luttes livrées pour le contrôle de cet environnement (Forsyth, 2003 ; Davis, 2016). L'étude des sciences et des sociétés a démontré que les choix sociaux et politiques s'insèrent systématiquement au sein des constructions de discours scientifiques (Latour, 1987). Un discours produit sur l'environnement est inévitablement influencé par la manière dont nous gérons ou souhaitons gérer cet environnement. Ceci mena Jasanoff à affiner le concept de coproduction initialement mis en avant par Latour. L'ordre social - l'ensemble des interactions et des relations de pouvoir entre humains - et l'ordre naturel - notre représentation de l'environnement - sont systématiquement co-produits (Jasanoff, 2004).

Plus spécifiquement, l'importance de l'institutionnalisation de la tenure de l'eau est perçue très différemment selon la position sociale des acteurs locaux et les modalités de leur tenure de la terre. La tenure de l'eau est plus institutionnalisée dans le cas de tout petits propriétaires qui se sentent davantage protégés par des règles collectives, tandis que les initiatives individuelles ne tenant pas compte des effets cumulatifs sur la nappe phréatique sont le modus operandi des agriculteurs qui disposent des moyens de forer des puits ou de faire appel à des technologies plus coûteuses de gestion de l'eau.

\section{Remerciements}

Tout au long de l'enquête de terrain, Rachad Antonius a bénéficié de l'appui gracieux de M. Elias Ghadban, Ph.D., diplômé en économie agricole de l'AUB (Université américaine de Beyrouth), et consultant pour diverses institutions internationales de développement. Très familier avec les enjeux, les personnes et les institutions impliquées dans les questions agricoles de la Beqaa, il a été un correspondant précieux qui a ouvert de nombreuses portes. Les auteurs remercient également l'Agence Nationale de la Recherche pour le financement du projet De terres et d'eaux ANR-12-AGRO- 
0002-01 qui a contribué à une partie de la recherche présentée dans cet article.

\section{Bibliographie}

Allan, J., 1997. Virtual water: a long term solution for water short Middle Eastern economies. Antonius, R, 1992. Irrigation et pouvoir social en Égypte. Thèse de doctorat, Université du Québec à Montréal.

Davis, D.K., 2016. The Arid Lands. MIT Press, Cambridge, MA. de Planhol, X., 1968. Les fondements géographiques de l'histoire de l'Islam. Flammarion, Paris. Forsyth, T., 2003. Critical Political Ecology: the politics of environmental science. Routledge, London.

Granott, A., 1952. The land system in Palestine. Eyre \& Spottiswoode, London.

Hamadeh,S., Ghosn,S, Rashid,G.,2005. Lebanon case study on Land tenure. Proceedings of the Regional workshop on Equitable Access to land and water resources. Beirut, Lebanon, 28-30 November.

Hodgson, S., 2016. Exploring the Concept of Water Tenure, Land and Water Discussion Papers. Food and Agriculture Organization of the United Nations, Rome.

Hoekstra, A.Y., Chapagain, A.K., Aldaya, M.M., Mekonnen, M.M., 2011. The Water Footprint Assessment Manual: Setting the Global Standard. Earthscan, London.

Hutteroth, W.-D., Abdulfattah, K., 1977. Historical Geography of Palestine, Transjordan and Southern Syria in the Late 16th Century. Erlangen Geographische Arbeiten, Erlangen.

Jasanoff, S., 2004. States of knowledge: the co-production of science and social order, International library of sociology. Routledge, London; New York.

Latour, B., 1987. Science in Action. Harvard University Press, Cambridge, Massachusetts.

Le Roy, É., 2011. La terre de l'autre une anthropologie des régimes d'appropriation foncière, Droit et société Série anthropologie. LGDJ-Lextenso éd., Paris.

Ministry of the Environment, Lebanon, 2001. State of the Environment Report, 2001, Chapter 8. Trottier, J., 2013. The social construction of water management at the intersection of international conflict The Case of Al Auja. Eurorient 161-181.

Trottier, J., Leblond, N., Garb, Y., 2019a. The Political Role of Date Palm Trees in the Jordan Valley: The Transformation of Palestinian Land and Water Tenure in Agriculture Made Invisible by Epistemic Violence, forthcoming.

Trottier, J., Perrier, J., 2018. Water driven Palestinian agricultural frontiers: the global ramifications of transforming local irrigation. Journal of Political Ecology 25, 292-311.

Trottier, J., Perrier, J., 2017. Challenging the Coproduction of Virtual Water and Palestinian Agriculture. Geoforum 87, 85-94. https://doi.org/10.1016/j.geoforum.2017.10.011

Trottier, J., Rondier, A., Perrier, J., 2019b. Palestinians and Donors Playing with Fire: 25 years of Water Projects in the West Bank. International Journal of Water Resources Development. https://doi.org/10.1080/07900627.2019.1617679

Universalis.fr (2019) Bekaa ou Beqaa, https://www.universalis.fr/encyclopedie/bekaa-beqaa/ consulté le 17 juin 2019. 